Published in final edited form as:

Transplant Proc. 2006 December ; 38(10): 3268-3270.

\title{
AN MHC CLASS II DISPARITY RAISES THE THRESHOLD FOR TOLERANCE INDUCTION IN PULMONARY ALLOGRAFTS IN MINIATURE SWINE1
}

\author{
Tsuyoshi Shoji, MD, $\mathrm{PhD}^{2}$, Hisashi Sahara, MD², Ashok Muniappan, MD², Dax A. Guenther, \\ MD ${ }^{2}$, Stuart L. Houser, MD ${ }^{2}$, Akshat C. Pujara ${ }^{2}$, Marjory A. Bravard ${ }^{2}$, John C. Wain, MD ${ }^{3}$, \\ David H. Sachs, MD ${ }^{2}$, Joren C. Madsen, MD, DPhil ${ }^{2,4}$, and James S. Allan, MD ${ }^{2,3,5}$ \\ 2 Transplantation Biology Research Center, Massachusetts General Hospital and Harvard Medical \\ School, Boston, MA 02114.
}

3 Division of Thoracic Surgery; Department of Surgery, Massachusetts General Hospital and Harvard Medical School, Boston, MA 02114.

4 Division of Cardiac Surgery; Department of Surgery, Massachusetts General Hospital and Harvard Medical School, Boston, MA 02114.

\section{Abstract}

Objectives-The mechanisms and treatment of chronic rejection in pulmonary allotransplantation remain elusive. We have induced robust tolerance to class I-disparate lung allografts in miniature swine using an intensive 12-day course of tacrolimus. Here, we test whether a tolerant state can be induced in swine receiving fully mismatched lung allografts.

Methods-Orthotopic left lung allografts were performed using MHC class I-disparate (Group 1: $\mathrm{n}=3$ ) or fully disparate (Group 2: $\mathrm{n}=6$ ) donors. The recipients received a 12-day post-operative course of tacrolimus (continuous IV infusion; target level $=35-50 \mathrm{ng} / \mathrm{ml}$ ) as their only immunosuppression.

Results-All swine in Group 1 maintained their grafts long term without developing any lesions of chronic rejection (> 497, > 432, and > 451 days). These recipients exhibited donor-specific hyporesponsiveness in cell-mediated lymphocytotoxity (CML) and mixed lymphocyte reaction (MLR) assays. In Group 2, 5 of the 6 recipients maintained their grafts long term (sacrificed on PODs $515,389,429,481$, and 438 with viable grafts). Isolated lesions of obliterative bronchiolitis (OB) were occasionally seen on biopsy, and donor-specific hyporesponsiveness on MLR and CML assays was consistently observed. The remaining recipient rejected its graft on POD 103 with histologic findings of OB.

Conclusions-We now report long-term graft acceptance without chronic immunosuppression in 5 of 6 recipients across a full MHC disparity, albeit with some evidence of OB. These data suggest that the class II disparity inherent in a fully mismatched transplant increases the requirement for tolerance induction.

\footnotetext{
${ }^{1}$ This work was supported in part by a research fellowship from the International Society for Heart and Lung Transplantation (TS), National Institute of Health grant 1RO1-HL67110 (JSA), and by awards from the American Surgical Association Foundation (JSA) and the Thoracic Surgery Foundation for Research and Education (HS).

5 Address correspondence to: James S. Allan, MD, Massachusetts General Hospital, Blake 1570, 55 Fruit Street, Boston, MA 02114. Fax: 978-745-9024, Phone: 617-724-1103, E-mail: jallan@partners.org.
} 


\section{INTRODUCTION}

As a result of advances in surgical techniques, immunosuppression, and postoperative management, lung transplantation has become an established therapeutic option for individuals with end-stage lung disease. The current 1-year actuarial survival rate following lung transplantation is approximately $75 \%$ (1). However, chronic lung allograft rejection, manifested principally by the histopathologic changes of obliterative bronchiolitis (OB), has limited the long-term success of lung transplantation. Almost 50\% of lung transplant recipients develop OB within 5 years of transplantation, and $\mathrm{OB}$ is currently the leading cause of graft loss and mortality after the first post-transplant year (1). The pathologic features of OB include scar formation and fibrosis in the small airways. However, the mechanisms and treatment of chronic rejection in pulmonary allotransplantation remain elusive. Using a strategy developed in our laboratory to induce tolerance across strong allogeneic barriers (2), we have previously induced robust tolerance to class I-disparate lung allografts in miniature swine with an intensive 12-day course of tacrolimus (3). Here, we test whether a tolerant state can be induced in swine receiving fully mismatched lung allografts.

\section{MATERIALS AND METHODS}

\section{Animals}

Transplant donors and recipients were selected from our herd of partially inbred miniature swine at 3-15 months of age. These swine have been inbred to homozygosity at the class I and class II MHC loci, as described previously (4). At present, three homozygous haplotypes are available for study. In addition, a number of intra-MHC recombinant haplotypes (derived from spontaneous recombination events) are also available $(4 ; 5)$. All animal care and procedures were in compliance with the "Principles of Animal Care" formulated by the National Society for Medical Research and the "Guide for the Care and Use of Laboratory Animals" prepared by the Institute of Laboratory Animal Resources and published by the National Institutes of Health (revised 1996).

\section{Experimental Design}

Eighteen swine were used in this study to conduct 9 orthotopic left lung allografts. 3 recipients received MHC class I-disparate lung allografts (Group 1) and 6 recipients received fully disparate lung allografts (Group 2), with a 12-day post-operative course of tacrolimus (continuous IV infusion; target level $=35-50 \mathrm{ng} / \mathrm{ml}$ ), as their only immunosuppression.

\section{Orthotopic Lung Transplantation}

Orthotopic left lung transplantation was performed as previously described (6). Briefly, under general anesthesia, the recipient's chest was entered through a left thoracotomy, and the hilar structures of the native lung were isolated. After heparinization $(300 \mathrm{U} / \mathrm{kg})$, the native lung was removed. The donor lung was approached through a median sternotomy. After heparinization $(300 \mathrm{U} / \mathrm{kg})$, the heart-lung block was topically cooled with iced saline and flushed in situ with 4 liters of cold Euro-Collins solution (Fresenius Medical Care AG, Bad Homburg, Germany) containing prostaglandin $\mathrm{E}_{1}(500 \mu \mathrm{g} / \mathrm{L})$. Immediately after harvesting, the left lung was surgically prepared and transplanted.

\section{Evaluation of Grafts}

In all experiments, lung allografts were monitored for rejection by a combination of chest radiography and serial open lung biopsy. The presence of high-grade histologic rejection and/ or the loss of graft aeration, compliance, or perfusion were the principal study endpoints. 
Immunologic status was evaluated by assays of cell-mediated lympholysis (CML) and the mixed lymphocyte reaction (MLR).

\section{RESULTS}

All swine in Group 1 maintained their grafts long term without developing any lesions of chronic rejection (> 497, $>432$, and $>451$ days). These recipients exhibited donor-specific hyporesponsiveness in cell-mediated lymphocytotoxity (CML) and mixed lymphocyte reaction (MLR) assays. In Group 2, 5 of the 6 recipients maintained their grafts long term (sacrificed on POD 515, 389, 429, 481, and 438 with viable grafts) (Fig. 1). Isolated lesions of obliterative bronchiolitis (OB) were occasionally seen on biopsy, and donor-specific hyporesponsiveness on MLR and CML assays was consistently observed. The remaining recipient rejected its graft on POD 103 with histologic findings of OB. In this animal, CML and MLR responses were seen on the day of rejection.

\section{CONCLUSIONS}

Pulmonary allograft tolerance has been consistently achieved across a class I-MHC barrier in this model. We now report long-term graft acceptance without chronic immunosuppression in 5 of 6 recipients across a full-MHC disparity, albeit with some evidence of OB. These data suggest that the class II disparity inherent in a fully mismatched transplant increases the requirement for tolerance induction. It is thus possible that peripheral mechanisms alone will be insufficient to permit the development of tolerance across some fully allogeneic barriers.

\section{References}

1. Trulock EP, Edwards LB, Taylor DO, Boucek MM, Mohacsi PJ, Keck BM, et al. The registry of the international society for heart and lung transplantation: twentieth official adult lung and heart-lung transplant report-2003. J Heart Lung Transplant 2003;22(6):625-635. [PubMed: 12821160]

2. Utsugi R, Barth RN, Lee RS, Kitamura H, LaMattina JC, Ambroz J, et al. Induction of transplantation tolerance with a short course of tacrolimus (FK506): I. Rapid and stable tolerance to two-haplotype fully mhc-mismatched kidney allografts in miniature swine. Transplantation 2001;71(10):1368-1379. [PubMed: 11391221]

3. Shoji T, Muniappan A, Guenther DA, Wain JC, Houser SL, Hoerbelt R, et al. Long-term acceptance of porcine pulmonary allografts without chronic rejection. Transplant Proc 2005;37(1):72-74. [PubMed: 15808551]

4. Sachs DH, Leight G, Cone J, Schwarz S, Stuart L, Rosenberg S. Transplantation in miniature swine. I. Fixation of the major histocompatibility complex. Transplantation 1976;22(6):559-567. [PubMed: 137560]

5. Pennington LR, Lunney JK, Sachs DH. Transplantation in miniature swine. VIII. Recombination within the major histocompatibility complex of miniature swine. Transplantation 1981;31(1):66-71. [PubMed: 6165111]

6. Allan JS, Wain JC, Schwarze ML, Houser SL, Benjamin LC, Madsen JC, et al. Modeling chronic lung allograft rejection in miniature swine. Transplantation 2002;73(3):447-453. [PubMed: 11884943] 

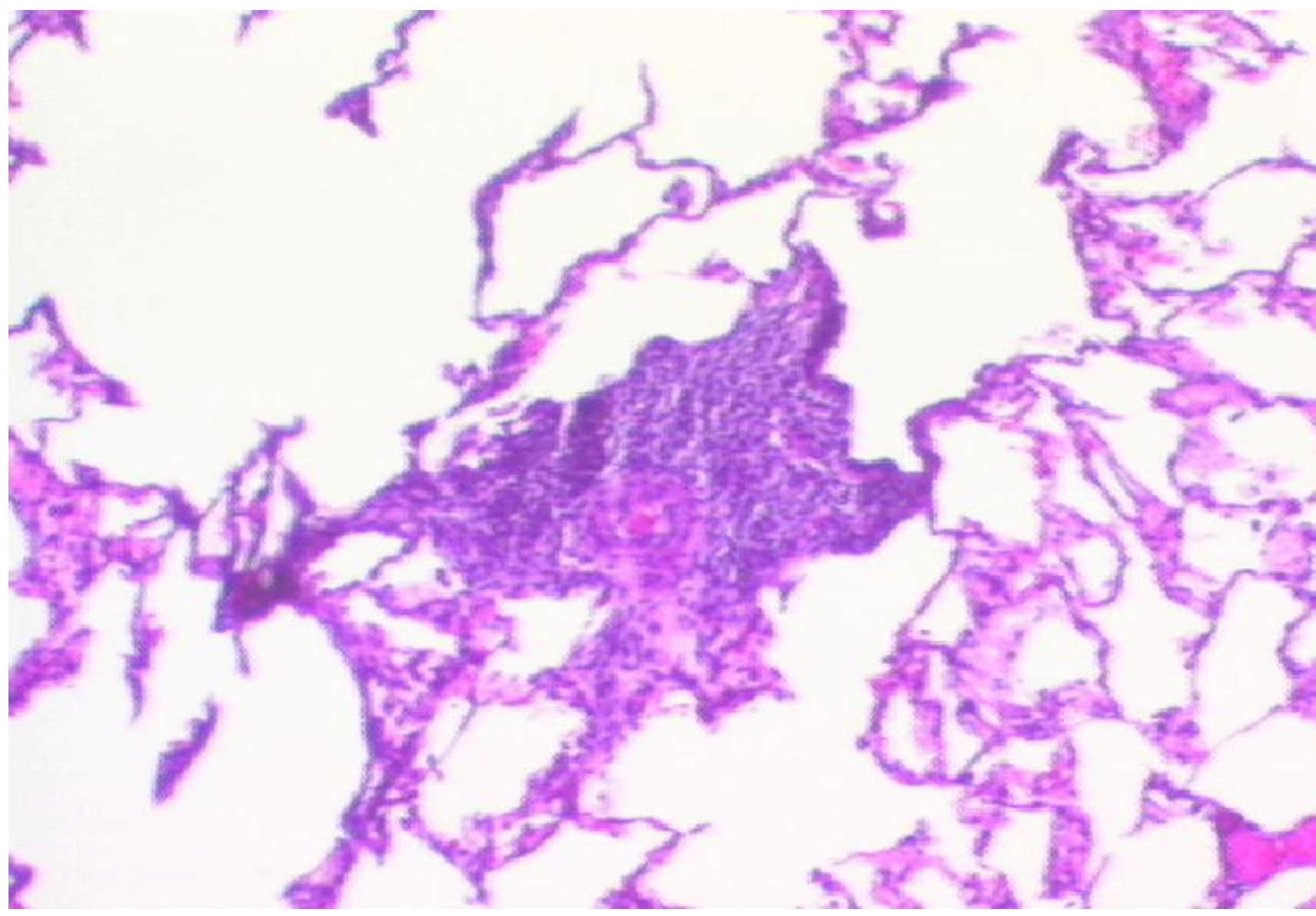


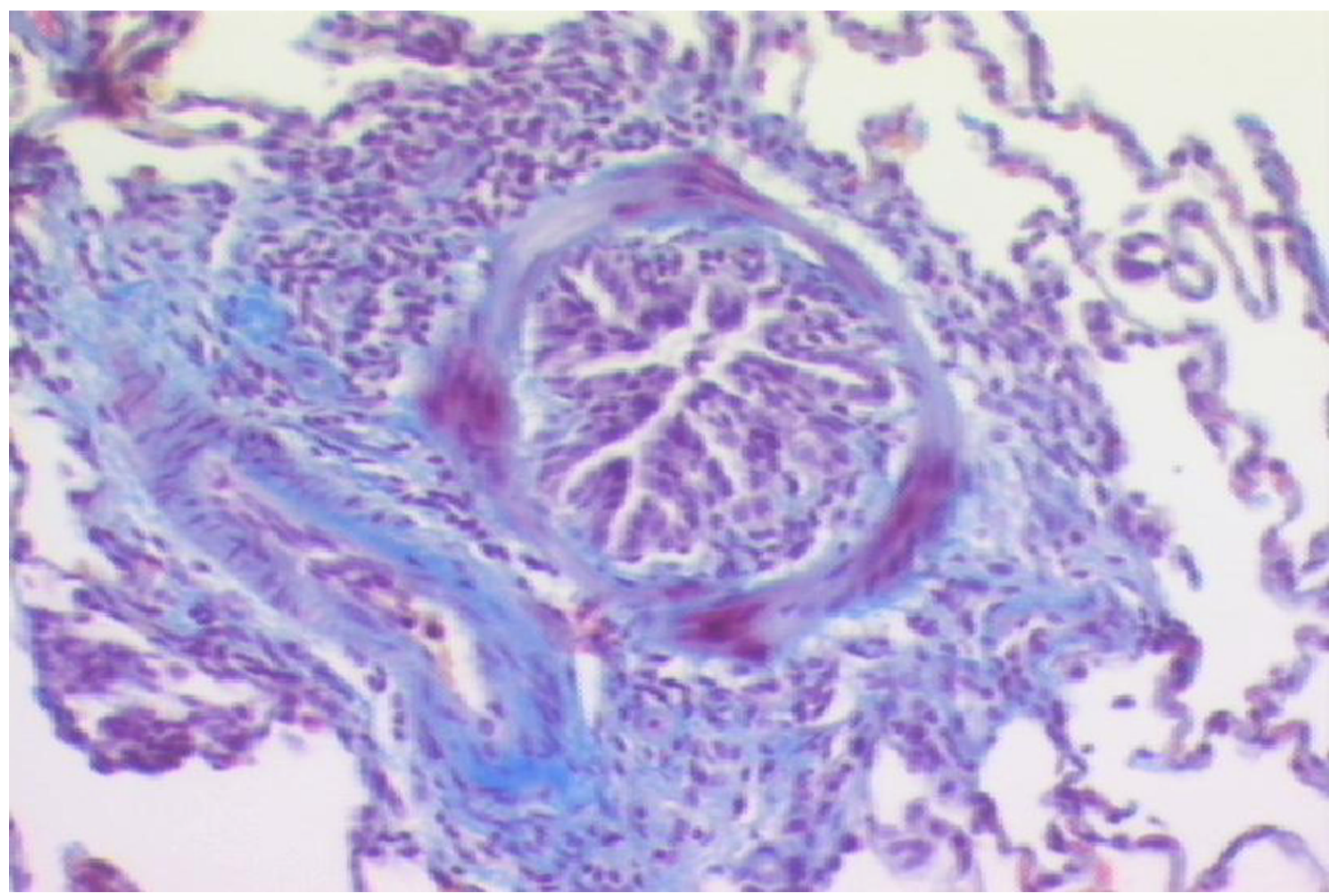

Figure 1.

Histological findings of fully mismatched lung allograft (Recipient \#15515; POD 347) Mild acute cellular rejection consistent with ISHLT grade $2 / 4$ acute rejection was seen (Figure 1a. H\&E stain, x100). No chronic rejection was observed (Figure 1b. Trichrome stain, x200). 